\title{
Haoyang $\mathrm{AN}^{\dagger}{ }^{\mathrm{a})}$, Nonmember and Jiageng $\mathrm{CHEN}^{\dagger \mathrm{b})}$, Member
}

SUMMARY The development of educational informatization makes data privacy particularly important in education. With society's development, the education system is complicated, and the result of education evaluation becomes more and more critical to students. The evaluation process of education must be justice and transparent. In recent years, the Onscreen Marking (OSM) system based on traditional cloud platforms has been widely used in various large-scale public examinations. However, due to the excessive concentration of power in the existing scheme, the mainstream marking process is not transparent, and there are hidden dangers of black-box operation, which will damage the fairness of the examination. In addition, issues related to data security and privacy are still considered to be severe challenges. This paper deals with the above problems by providing secure and private transactions in a distributed OSM assuming the semitrusted examination center. We have implemented a proof-of-concept for a consortium blockchain-based OSM in a privacy-preserving and auditable manner, enabling markers to mark on the distributed ledger anonymously. We have proposed a distributed OSM system in high-level, which provides theoretical support for the fair evaluation process of education informatization. It has particular theoretical and application value for education combined with blockchain.

key words: onscreen marking, consortium blockchain, privacy-preserving, examination, auditable

\section{Introduction}

The continuous development of emerging technologies such as cloud computing, artificial intelligence, and blockchain has changed the traditional way of data collection and transmission of human beings, leading to qualitative data storage and computing changes and providing a powerful driver for educational innovation. It provides reliable technical support for the reform of education informatization. The powerful capabilities of blockchain technology, such as nontampering, transparency and traceability, and automatic contract execution, have become the driving force for integrating various industries. The advancement of education informatization has made data privacy particularly important in the education field. Various online education platforms have collected a large amount of user data, and through data analysis, students' privacy can be explored relatively

Manuscript received April 8, 2021.

Manuscript revised November 19, 2021.

Manuscript publicized December 6, 2021.

${ }^{\dagger}$ The authors are with the School of Computer, Central China Normal University, No.152 Luoyu Road, Wuhan, Hubei, 430079, China.

${ }^{*}$ An extended abstract of this paper appears at the 2021 IEEE Conference on Dependable and Secure Computing (IEEE DSC 2021). This is the full version.

a)E-mail: haoyang.an@outlook.com

b) E-mail: chinkako@gmail.com (Corresponding author)

DOI: $10.1587 /$ transinf.2021BCP0004 accurately. With the development of the social economy, the educational system has become larger and larger, and the results of the educational evaluation are becoming more and more critical to students. The evaluation process of educational evaluation needs to be fair, just, and open. The existing traditional OSM may tamper with data due to the excessive power of the center administrator. In this context, we began to explore the deep integration of blockchain and education scenarios.

Recently, Onscreen Marking (OSM) system [1] is a standard electronic scoring system for examinations. It uses an electronic scanner to scan the answer sheet submitted for the exam and processes the data by a computer. It can support high concurrent access and can monitor the marking process and progress in real-time. The examination center will hide the students' sensitive information, divide candidatess' answers according to the questions, and randomly assign them to markers for scoring.

OSM is now the most popular examination marking method in the world. There are tens of thousands of largescale public examinations every year in the world. For example, millions of candidates take part in China's National College English Test (CET-4/CET-6) every year. Under the support of the Hong Kong government [2], 13 assessment centers provide excellent accessibility for markers are available for the 2015 Hong Kong Diploma of Secondary Education [3].

Many data service providers provide specialized examination marking services, such as DRS Data Services Limited [4] and RM Results [5]. According to the DRS report, up to 2015, Zimbabwe Schools Examinations Council [6] has scanned over 20 million sheets. Jawaharlal Nehru Technological University [7] with over 300,000 students and around 3.7 million scripts a year has successfully implemented an automated online marking system through DRS. The processing workload is undoubtedly immense, but now with the OSM system, inspection results can be delivered faster and more accurately than ever before. RM Results provide digital assessment solutions that support lifelong learning. Their products are now widely used in the Institute of Chartered Accountants of Nigeria's [8], the New Zealand Qualifications Authority (NZQA) [9], and the Association of Chartered Certified Accountants (ACCA) [10].

Using traditional marking methods, scripts may be transmitted multiple times, for example, from the examination center to markers, auditors, candidates. The more people deal with it, the more likely there is to cheat. OSM 
dramatically simplifies the number of transfers of scripts, ensures its physical security, and can significantly reduce the risk of other potential security vulnerabilities. OSM's anonymous process reduces the markers' conscious or unconscious bias and promotes the fairness of the exam. Despite the popularity of OSM, there are still many security risks with this solution. For example, the current OSM is based on a fully centralized service, making the center too powerful. Once the center is compromised, the fairness of the exam will be badly damaged. Besides, the entire process of marking is not open to the candidates, and candidates cannot obtain specific information for marking, nor can they trace their grades.

The decentralized, non-tamperable features of blockchain provide new solutions for data security and privacy protection. However, issues such as data sharing on the ledger have brought new problems and challenges to privacy. Privacy-preserving blockchain has become a research hotspot. Existing innovative research mostly come from the field of cryptocurrency and electronic health record (EHR), which include stealth address [11], [12], zero-knowledge proofs [13], [14], ring signatures [12], attribute-based encryption [15]-[17] and so on. However, there are problems such as insufficient privacy protection, lack of balance between privacy protection and security auditing.

Most of the industrial applications based on blockchain are concentrated in the consortium blockchain. Compared with public blockchains, the identity of participants can be controlled to a certain extent. The Hyperledger Fabric [18] framework designed by IBM has been widely used. It realizes the anonymity and untraceability of transaction initiators. In the latest v2.0 version, private data protection is enhanced by sharing and verifying private data. However, even in this way, the sender's identity and the content of the transaction cannot be privacy protected, nor can it provide fine-grained regulatory control over privacy-protected data.

\subsection{Our Contributions}

Our contributions are mainly described in the following three aspects:

1) We designed an ExamChain based on consortium blockchain to achieve security, fair, transparent, and privacy-preserving marking processes in examinations. It provides complete privacy protection for candidates' identity, question ID, answers content, marking scores, and markers' identity based on common assumptions.

2) We provided a credible distributed framework for the research of onscreen marking systems from the view of comprehensive data security and privacy protection. According to the real evaluation scenario, we have defined specific privacy rules.

3) Considering the massive amount of data in large-scale public examinations, different from the conference version [19], by using conditional proxy re-encryption (C-PRE) technology, we have added a semi-honest third-party cloud server closer to the reality to reduce EC storage pressure.

\subsection{Organization}

The organization of the remaining sections is as follows. Section 2 introduces the background of related works. Section 3 introduces the preliminaries used in protocol. Section 4 presents the overview of the ExamChain, which describes the system model, marking workflow, and security model. Section 5 presents our concrete protocol from four aspects: candidate and question privacy, candidate and question ID authentication, marking scores privacy, and marker identity privacy. Section 6 analyzes the performance of our system. Finally, Sect. 7 concludes the paper.

\section{Related Works}

\subsection{Onscreen Marking System}

Onscreen Marking (OSM) system [1] has been widely used in many educational institutions. The process of OSM as shown in Fig. 1. The exam requires a unique barcode label to be attached to the answer sheet to display the candidate's information and the script. The examination center scans these scripts uniformly, stores the resulting images in a secure database, and then sends them to different marking markers. It can also send a script to multiple markers for marking multiple times, which improves objectivity. The examination center will monitor the marking progress and accuracy in real-time and make adjustments on time. OSM further improves the accuracy, safety, and efficiency of the exam marking process. More and more examination institutions began to adopt this mode of marking.

Compared with the manual process, the main benefits of OSM are the enhancement of security, marking quality, efficiency, accuracy, and data availability. One-time scanning eliminates the physical handling of the answer sheet. The examination center can monitor the marking process in real-time, find problems, and deal with them in time. Examiner can check the answer sheet from any location. Final result processing is accurate and completed in a quick time. On the other hand, there are issues such as marker acceptance, the reliability of the examination center, etc. Markers need to travel to mark at specific locations and at specific times, which is inconvenient. The marking process information is opaque, and there is a possibility of a black-box operation damaging fairness. The examination center may be subject to malicious attacks resulting in data tampering or unauthorized access. Data sharing lacks complete privacy protection.

\subsection{Consortium Blockchain}

Blockchain is a chain-type data structure that combines blocks in chronological order and is a cryptographically guaranteed non-tamperable and unforgeable distributed 


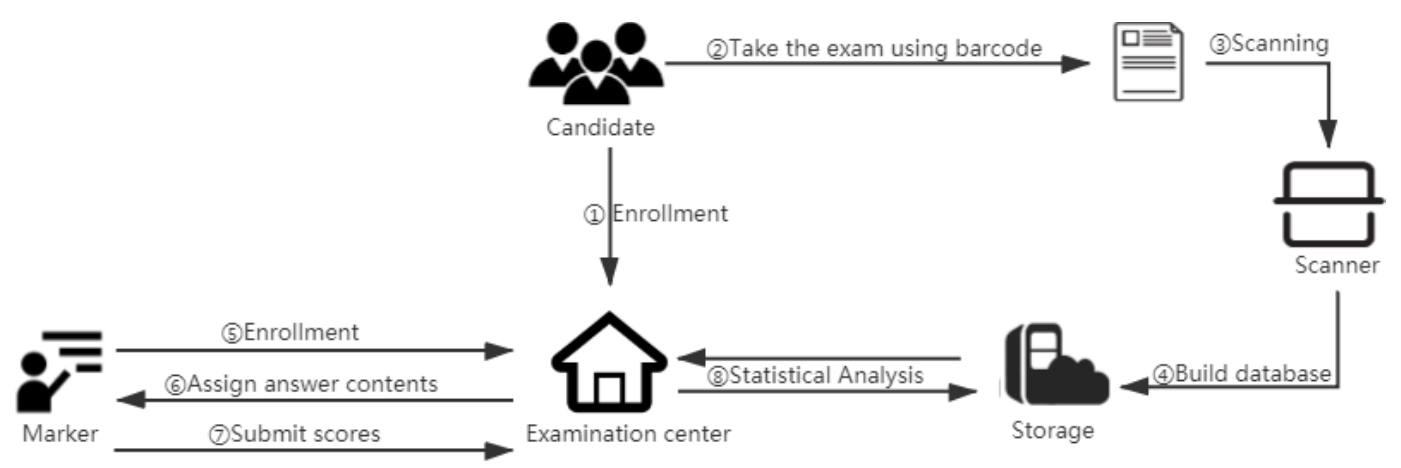

Fig. 1 Onscreen marking system.

ledger. When the access control of the blockchain is managed by a membership service provider, a distributed storage within an organization is realized, which is called a consortium blockchain. Different from the public chain, in the consortium blockchain represented by the HyperLedger Fabric [18] blockchain platform, peers do not trust each other, but the authenticity and accuracy of other peers' accounting can be confirmed by means of information verification in the consensus algorithm to generate a distributed semidecentralized ledger.

Many researches on blockchain application are in cryptocurrency and electronic health record. However, they cannot be applied to the scenario of the examination. For cryptocurrency, Bitcoin [11] is the first mature application of the blockchain. It uses pseudonyms for transactions. The private key corresponding to its address is the only proof of ownership of Bitcoin. Therefore, Bitcoin uses pseudonyms to achieve a certain degree of anonymity. However, In 2013, Androulaki et al. [20] proposed a method of mining Bitcoin addresses. If a transaction has two outputs, one of which is the address that has already appeared, and the other is the new address, that is, the change address and input of the transaction. The address is held by the same user. They built a simulated cryptocurrency usage environment, collected user usage records, and analyzed the real identities of about $40 \%$ of users. In 2013, Saberhagen et al. [21] proposed the CryptoNote protocol based on ring signature, improved linkable ring signatures to protect the privacy of the sender, and used a one-time public-private key pair to protect the privacy of the receiver. Monero [12] uses CryptoNote as the underlying framework to protect user privacy, but the ring size is increasing with the number of users, resulting in lower and lower efficiency. Zcash [13] used zero-knowledge proof (zk-SNARK) to achieve user privacy with low efficiency. Since their privacy protection is completely anonymous and cannot be audited, they cannot prevent criminal acts such as money laundering [22]. PAChain [23] presented a private, authenticated, and auditable consortium blockchain, which provided complete privacy protection from three aspects of sender privacy, receiver privacy, and transaction amount privacy. But they are generally based on the UTXO model, which is mainly designed for the financial system. The transaction content is the amount of money that needs to be protected from double-spending attacks.

For electronic health record [24], Sun et al. [25] proposed a blockchain-based EHR that uses decentralizing attribute-based signature for access control, and stores a large amount of data in a third-party trusted cloud as an off-chain database. However, a fully trusted cloud is unrealistic, and patients have no right to control their data. Wang et al. [16] used attribute-based encryption (ABE) to encrypt the file encryption symmetric key. When the data visitor's attribute meets the access policy defined by the patient, the decryption information is obtained and the file is downloaded and decrypted from InterPlanetary File System (IPFS). However, IPFS itself does not have sufficient privacy protection design. Once the symmetric secret-key is leaked, ABE will become useless. Liu et al. [17] used ciphertext policy attribute-based encryption (CP-ABE) access control to store patient data in the cloud, reducing the storage burden of the blockchain. The data visitor extracts the signed data from the cloud and verifies the validity and integrity of the data through the blockchain. These blockchain solutions for EHR have certain reference significance, but they cannot be directly applied to the examination marking process. They lack a complete privacy protection design, which will bring many new problems. For instance, the data producer should not fully control the access control rights of the data which should be handled by the examination center.

In Hyperledger Fabric [18], it cannot achieve finegrained access control and cannot comprehensively protect transaction privacy. Transactions are handled by different channels and users can only view transactions in their channel. Therefore, privacy is maintained by the system's access control policy.

\section{The Preliminaries}

In this section, we introduce several technical security related primitives that will be used in our solutions.

\subsection{Bilinear Groups}

Bilinear pairings is a mathematical mapping relationship, which serves as a construction tool for cryptographic algorithms. In modern public-key cryptography, bilinear 


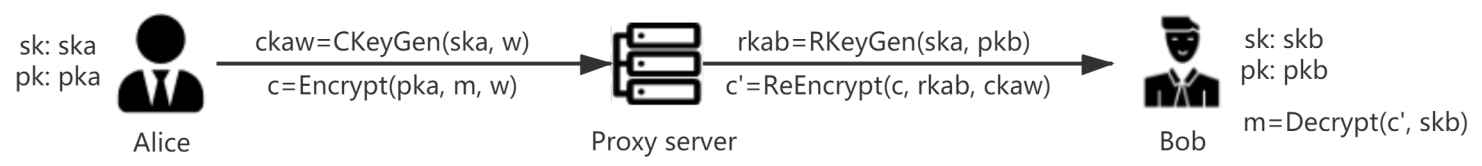

Fig. 2 Conditional proxy re-encryption.

pairing provides many cryptographic functions to solve more complex problems. Elliptic curve pairings dramatically improves the efficiency of pairing-based cryptosystems, and the application of cryptography based on bilinear pairing has become a research hotspot. Bilinear groups are a set of three abstract algebraic groups, together with a deterministic function, called a bilinear map. $\mathcal{G}$ is an algorithm, which takes as input a security parameter $\lambda$ and outputs a tuple $\left(p, \mathbb{G}_{1}, \mathbb{G}_{2}, \mathbb{G}_{T}, \mathbb{G}_{T}^{\prime}, \hat{e}, e\right)$ where $\mathbb{G}_{1}, \mathbb{G}_{2}, \mathbb{G}_{T}$, $\mathbb{G}_{T}^{\prime}$ are multiplicative cyclic groups with prime order $p$, and $e: \mathbb{G}_{1} \times \mathbb{G}_{1} \rightarrow \mathbb{G}_{T}^{\prime}, \hat{e}: \mathbb{G}_{1} \times \mathbb{G}_{2} \rightarrow \mathbb{G}_{T}$ are two map, which have the following properties:

- Bilinearity: For $\forall g_{1}, g_{2} \in \mathbb{G}_{1}, \hat{g} \in \mathbb{G}_{2}$ and $\forall a, b \in \mathbb{Z}_{p}$, $\hat{e}\left(g_{1}^{a}, \hat{g}^{b}\right)=\hat{e}\left(g_{1}, \hat{g}\right)^{a b}, e\left(g_{1}^{a}, g_{2}^{b}\right)=e\left(g_{1}, g_{2}\right)^{a b}$.

- Non-degeneracy: There exists $g_{1}, g_{2} \in \mathbb{G}_{1}, \hat{g} \in \mathbb{G}_{2}$ such that $\hat{e}\left(g_{1}, \hat{g}\right) \neq 1, e\left(g_{1}, g_{2}\right) \neq 1$.

- Computability: There exists an efficient algorithm to compute $\hat{e}\left(g_{1}, \hat{g}\right)$ for $\forall g_{1} \in \mathbb{G}_{1}, \hat{g} \in \mathbb{G}_{2}$, and $e\left(g_{1}, g_{2}\right)$ for $\forall g_{1}, g_{2} \in \mathbb{G}_{1}$.

\subsection{ElGamal Encryption}

In cryptography, ElGamal encryption algorithm is an asymmetric encryption algorithm based on Diffie-Hellman key exchange. It has been widely investigated to be secure and efficient. More importantly, it is flexible which can be easily connected to other more advanced protocols. We will in our instantiation use ElGamal encryption (over setup $g k$ ) described as follows:

- $\operatorname{PKEGen}(g k): d k \leftarrow \mathbb{Z}_{q}^{*} ; p k:=g^{d k} ; \operatorname{Return}(p k, d k)$.

- $\operatorname{Enc}(p k, m)$ : If $p k \notin \mathbb{G}_{1}^{*}$ or $m \notin \mathbb{G}_{1}$ return $\perp$. $r \leftarrow \mathbb{Z}_{q}$; Return $c:=\left(p k^{r}, g^{r} m\right)$.

- $\operatorname{Dec}(d k, c)$ : If $d k \notin \mathbb{Z}_{q}^{*}$ or $c \notin \mathbb{G}_{1}^{2}$ return $\perp$. Return $m:=v u^{-\frac{1}{d k}}$.

\subsection{Boneh-Boyen Signature (BBS)}

Digital signature is a digital string that can only be generated by the sender of the message, and this digital string is also an effective proof of the authenticity of the message sent by the sender. Meanwhile, anyone with the public key can efficiently verify its legality. Camenisch et al. [26] proposed a signature-based range proof. The signer's secret key is $x \in$ $\mathbb{Z}_{p}$, the corresponding public key is $Y=g^{x}$. The signature on a message $m$ is $\sigma=g^{\frac{1}{x+m}}$; verification is done by checking that $e\left(\sigma, Y \cdot g^{m}\right)=e(g, g)$. On input the bilinear group $\hat{e}(g, \hat{g})$

- $\operatorname{Sign}(s k, m): \sigma=\hat{g}^{\frac{1}{k+m}}$, output $\sigma$.

- Verifysign $(p k, \sigma, m)$ : if $\hat{e}\left(p k \cdot g^{m}, \sigma\right)=e(g, \hat{g})$, output true, otherwise output false.

\subsection{Signature-Based Range Proof}

Range proof uses zero-knowledge proof and other technologies to prove that the secret is within the compliance scope without revealing the specific secret content. Camenisch et al. [27] proposed a range proof based on Boneh-Boyen signature set. An authority sign all elements in $[0, R]$ to get set $\phi$. Then the prover wants to prove the secret $x$ in $\phi$ without revealing $x$ nor the signature to ensure a valid secret.

\subsection{Conditional Proxy Re-Encryption}

Proxy re-encryption is to entrust a semi-honest agent to transform the ciphertext encrypted by its public key into a new ciphertext that can be decrypted by the other party's secret key, thereby realizing data sharing. Weng et al. [28] first proposed a CCA secure conditional proxy re-encryption scheme under the 3-Quotient Bilinear Diffie-Hellman (3QBDH) assumption in 2009. For briefly, a C-PRE scheme involves three parties: a data owner (Alice), a proxy server, and a data receiver (Bob) as in Fig. 2. The sender uses Alice's public keys $p k a$ and $w$ to encrypt the plaintext sent to Bob whose condition is $w$. In order to authorize Bob to decrypt the encryption associated with $w$, Alice provides the agent with a partial re-encryption key $r k a b$ and a condition key ckaw corresponding to the condition $w$. These two keys constitute a secret trap door used by the agent to perform ciphertext conversion. The agent cannot convert those ciphertexts whose corresponding condition keys are not available. Therefore, Alice can flexibly control delegation by appropriately releasing the condition key.

\section{ExamChain Overview}

\subsection{System Model}

We extended the Hyperledger Fabric [18] system model. A client submits a transaction (Tx) proposal to the endorser, and the endorser simulates the execution of the transaction and endorses it. Only the endorsed Tx can be submitted to the orderers. Sequentially, the orderer broadcast Txs in order, and the committing peers write block of Txs with valid endorsement. Each block contains a completely ordered set of Tx. The distributed ledger can be accessed by any node. We define seven types of nodes in ExamChain (Fig. 3):

- Candidate (Client): A client can access the ledger in the system to verify his/her marking records of their scripts. 


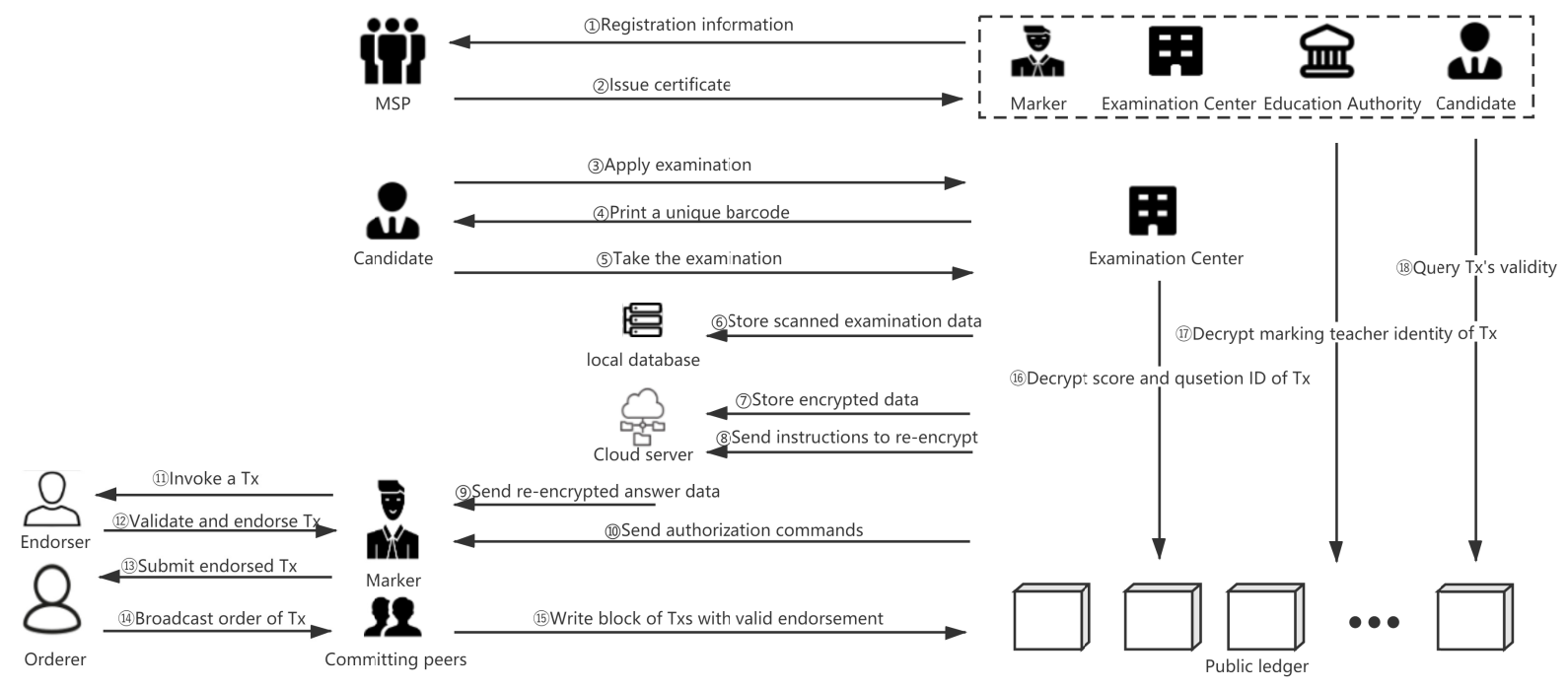

Fig.3 ExamChain overview: we use the blockchain stealth address technique for candidate privacy. We use Boneh-Boyen signature-based range proof for transaction score privacy. We use hash and reserved data sharing for question ID and answer content privacy. We use anonymous credentials for marker privacy. We use authentication protocol and proxy re-encryption for candidate and question ID authentication.

- Marker (Client): A client initializes and submits a Tx to endorsers and broadcasts Tx proposals with the endorsement to orderers.

- Peer: A peer can be the endorser and committing peers concurrently. Endorsers check the validity of Txs. Committing peers commits Txs and maintains the distributed ledger.

- Orderer: Orderers order Txs and run consensus algorithm.

- Examination center (EC): The examination center can recover the question ID and the Tx score of any transaction.

- Education authority (EA): The education authority can recover the Marker's identity of any transaction.

- Membership Service Provider (MSP): MSP issues certificates to all legal participants. An only authorized party can create Txs.

\subsection{Marking Workflow}

1. The four organizations (Marker, Examination center, Education authority, Candidate) submit registration information to MSP to join the system. The marker needs to register with his/her information to the EA and EC.

2. The MSP issue certificate for every participant.

3. A candidate registers with his/her information to the $\mathrm{EC}$, and applies for the examination.

4. The EC stores the candidate's long-term public key and combines the candidate's information with examination information to export a unique examination paper ID and print it as a barcode.

5. Candidates take the examination by pasting the barcode on the examination paper.

6. The EC scans examination paper, split pictures, and builds a local database.

7. The EC encrypts the answer content data and sends it to a cloud server.

8. When the EC needs to distribute answer content to markers. The EC sends instructions to the cloud server to transform ciphertext to indicated marker.

9. The cloud server sends re-encrypted answer data to an indicated marker.

10. The EC sends authorization commands to the indicated marker.

11. The marker obtains answer content, marks the score, and creates a signed $T x$ and sends it to endorsers.

12. Each endorser simulates execution and verifies Tx generates endorsement signature for Tx, and sends it back to the marker.

13. Marker submits the endorsed Tx to orderers.

14. The orderer broadcasts the ordered Txs blocks to all peers.

15. The committing peer checks whether the Tx complies with the above process and submits compliant Txs for a public ledger record.

16. The EC recoveries the question ID and the Tx score. Record all the Txs on the database and give final scores according to the specific marking rules. Note that multiple different markers may score the same question.

17. The EA can recoveries the marker identity from the public ledger when necessary.

18. Candidates can verify the source of their scores by accessing the public ledger.

\subsection{Security Model}

- Candidate privacy: No one can learn candidates' sensitive information, except for the EC. 
- Marking score privacy: No output score is outside the range. No one can learn the output score except the EC and the corresponding candidate.

- Question ID and answer content privacy: Only the EC can compute Question ID and answer content.

- Marker privacy: No adversary can mark without a credential, even with colluding EA. No one can learn the identity of the marker, except the EA.

- Candidate and question ID authentication: No adversary can propose a candidate's identity and question ID issued from others except the EC, even with colluding markers. Even if cloud servers that have neither partial re-encryption keys nor conditional keys collude with markers, they cannot compromise the security of the EC. The cloud server possessing part of the reencryption key and the condition key at the same time will not harm the security of the EC or the marker.

\section{Our Protocol}

\subsection{Candidate and Question Privacy}

\subsubsection{System Model}

A candidate registers the identity in MSP to obtain a certificate. Then, he/she apply to the EC for an exam with his/her sensitive information. After the examination, the EC will scan answer sheets for data processing and establish a secure local database to store candidates' information as following: candidate information info $o_{c a n}$, examination information info $o_{\text {exam }}$, candidate's long-term public key $c p k$, examination paper ID paperID, answer content q content $_{n}$ of each question, question ID qid, ( $n$ is the question number). It will never send candidates' sensitive information during the whole marking process, except their one-time public keys derived from their long-term public keys. To prevent tampering with the data after scanning the answer sheet, we derive the 16 bytes qid in the following way: Suppose $H$ is a collision-resistant hash function like SHA-256.

$$
\text { qid }=H\left(c p k, \text { paperID }, n, q \text { content }_{n}\right)
$$

Only the EC knows all the information in Eq. (1) and can produce qid because of the hash function's one-way property. As a result, qid can be learned by others, which does not comprise candidate and question privacy.

- (param, ask $\left.k_{e a}, a s k_{e c}\right) \leftarrow$ GlobalSetup $\left(1^{\lambda}, R\right)$. On input a security parameter $1^{\lambda}$ and the range parameter $R$. It outputs both the EA and the EC secret key askea $a s k_{e c}$, and the system parameter param. For simplicity, we assume that the param is implicitly included in the input of the remaining algorithms.

- (csk, $c p k) \leftarrow$ CanKeyGen(). The candidate outputs his secret key $c s k$ and public key $c p k$.

- $(\operatorname{cotp} k, \cot R) \leftarrow$ CanOTPkGen $(c p k)$. On input a public key $c p k$, it outputs a one-time public key cotpk and a randomness $\cot R$.
- cotsk $/ \perp \leftarrow$ CanOTSkGen $(\operatorname{cotp} k, \cot R, c s k)$. On input a public key cotpk, a randomness $\cot R$ and secret key $c s k$, it outputs a one-time secret key cotsk or $\perp$ if cotpk is not related to $c s k$.

- $(\cot p k, \cot R) \leftarrow$ CandidatePrivacy $(c p k)$. On input a candidate's public key $c p k$, it outputs cotpk and $\cot R$.

\subsubsection{Security Property}

Only the EC can compute Question ID and answer content if the collision-resistant assumption holds in $H$. The inputs of $H$ are created by the EC and never send to others. Stealth address [12] hide the candidate's real identity. A candidate is anonymous except for the EC if the Decision Diffie-Hellman (DDH) assumption holds in $\mathbb{G}_{1}$ in the random oracle (RO) model. A candidate submits his/her sensitive information to the EC at first to verify the candidate's identity. During the whole process, the EC never sends the candidate's sensitive information to anyone.

\subsubsection{Proof Sketch}

Observe that $\cot R=g^{\text {cotr }}, \operatorname{cotpk}=Y_{1} g^{H^{\prime}\left(Y_{2}^{\text {cotr }}\right)}$. Assume $H^{\prime}$ is a secure hash function and the DDH holds in $\mathbb{G}_{1}$. cotpk, $\cot R$ does not provide information of $c p k$. Hence, the adversary has no advantage of winning the anonymity game if the DDH holds in $\mathbb{G}_{1}$ in RO.

\subsubsection{Construction Details}

To avoid markers to learn the candidates' identity, stealth address [12] provide a method to hidden a candidate's longterm public key. The EC computes and stores condidates' one-time public key by their long-term public key. Then the EC interacts with markers with candidates' one-time public key.

* GlobalSetup On input a security parameter $1^{\lambda}$ and the range parameter $R=u^{l}$, it generates the bilinear group by $\left(p, \mathbb{G}_{1}, \mathbb{G}_{2}, \mathbb{G}_{T}, \mathbb{G}_{T}^{\prime}, e, \hat{e}\right) \leftarrow \mathcal{G}\left(1^{\lambda}\right) . \mathbb{G}_{1}, \mathbb{G}_{2}$ and $\mathbb{G}_{T}$ are three cyclic groups with prime order $p$, and $e, \hat{e}$ are two bilinear pairing $e: \mathbb{G}_{1} \times \mathbb{G}_{1} \rightarrow \mathbb{G}_{T}^{\prime}, \hat{e}: \mathbb{G}_{1} \times \mathbb{G}_{2} \rightarrow \mathbb{G}_{T}$. It picks some random generators $g, g_{0}, g_{1}, u_{1}, h_{s} \in \mathbb{G}_{1}$, $\hat{g}, \hat{g}_{2} \in \mathbb{G}_{2}$, and $x \in \mathbb{Z}_{p}$. It computes $\hat{Y}=\hat{g}^{x}, A_{i}=g^{\frac{1}{x+i}}$ for $i \in[0, u-1]$. Suppose there are seven collision resistant hash function: $H, H^{\prime} H_{1}, H_{2}, H_{3}, H_{4}$ and $H_{5}$ such that $H:\{0,1\}^{*} \rightarrow \mathbb{Z}_{p}, H^{\prime}: \mathbb{G}_{1} \rightarrow \mathbb{Z}_{p}, H_{1}:\{0,1\}^{*} \rightarrow \mathbb{Z}_{p}^{*}, H_{2}:$ $\mathbb{G}_{T}^{\prime} \rightarrow\{0,1\}^{l_{0}+l_{1}}, H_{3}:\{0,1\}^{*} \rightarrow \mathbb{G}_{1}, H_{4}: \mathbb{G}_{T}^{\prime} \rightarrow\{0,1\}^{l_{0}+l_{1}}$ and $H_{5}: \mathbb{G}_{1} \times \mathbb{G}_{1} \times\{0,1\}^{l_{0}+l_{1}} \rightarrow \mathbb{Z}_{p}^{*}$. Here $l_{0}$ and $l_{1}$ are determined by the security parameter, and the content space is $\{0,1\}^{l_{0}}$.

Suppose the EC picks a random secret key ask $k_{e c} \in \mathbb{Z}_{p}$ and outputs its public key $h_{e c}=g^{a s k_{e c}}, h_{e c}^{\prime}=\left(h_{e c}, g_{1}^{1 / a s k_{e c}}\right)$. Suppose the EA picks a random secret key $a s k_{e a} \in \mathbb{Z}_{p}$ and outputs its public key $h_{e a}=g^{a s k_{e a}}$. The global parameters are param $=\left(p, \mathbb{G}_{1}, \mathbb{G}_{2}, \mathbb{G}_{T}, e(g, g), \hat{e}(g, \hat{g}), g\right.$, $g_{0}, g_{1}, u_{1}, h_{s}, h_{e c}, h_{e c}^{\prime}, h_{e a}, \hat{g}_{2}, H, H^{\prime}, H_{1}, \cdots, H_{5}, \hat{Y}, A_{0}, \ldots$, 
$\left.A_{u-1}, u, l\right)$.

* CanKeyGen A candidate randomly picks $x_{1}, x_{2} \in \mathbb{Z}_{p}$ as his/her long-term secret key and computes public key $Y_{1}=g^{x_{1}}, Y_{2}=g^{x_{2}}$. Output key pair $c s k=\left(x_{1}, x_{2}\right)$, $c p k=\left(Y_{1}, Y_{2}\right)$.

* CanOTPkGen On input $c p k=\left(Y_{1}, Y_{2}\right)$, it randomly picks cotr $\in \mathbb{Z}_{p}$ and outputs $\cot R=g^{\text {cotr }}$, cotpk $=$ $Y_{1} g^{H^{\prime}\left(Y_{2}^{\text {cotr }}\right)}$.

* CanOTSkGen On input cotpk, cotR, csk $=\left(x_{1}, x_{2}\right)$, the recipient computes and outputs a one-time secret key cotsk $=x_{1}+H^{\prime}\left(\right.$ ot $\left.^{x_{2}}\right)$.

* CandidatePrivacy A candidate runs $(c s k, c p k) \leftarrow$ CanKeyGen(), and sends his/her long-term public key $c p k$ to the EC. The EC runs $(\operatorname{cotpk}, \cot R) \leftarrow$ CanOTPkGen $(c p k)$, and sends cotpk, $\cot R$ to the candidate. The candidate runs cotsk $/ \perp \leftarrow$ CanOTSkGen (cotpk, cotR, $c s k)$ to get his/her one-time secret key.

EC never sends any sensitive information about candidates to others except one-time public key cotpk to indicated markers during the whole process. The onetime public key hides the true identity of the candidate, which protects their privacy.

\subsection{Candidate and Question ID Authentication}

\subsubsection{System Model}

- $(m s k, m p k) \leftarrow$ MarKeyGen(). A marker outputs his/her long-term secret key and public key $m s k, m p k$.

- $m r k \leftarrow$ RencKeyGen $\left(a s k_{e c}, m p k\right)$. EC takes its secret key $a s k_{e c}$ and the public key $m p k$ indicating the marker as input. It outputs part of the re-encryption key $m r k$.

- $m c k_{w} \leftarrow$ CondKeyGen $\left(a s k_{e c}, w\right)$ EC takes its key $a s k_{e c}$ and condition $w$ as input. The condition $w$ means EC's condition for batch distribution of questions based on access control. It outputs the condition key $m c k_{w}$.

- $C T_{e c} \leftarrow \operatorname{ProxyEncrypt~}\left(h_{e c}^{\prime}, q c o n t e n t, w\right)$. EC takes the public key $h_{e c}^{\prime}$, a set of local plaintext answer content qcontent and the condition $w$ with relevant metadata as input. It encrypts the ciphertext $C T_{\text {server }}$ associated with condition $w$ under the public key $h_{e c}^{\prime}$ of the EC.

$-C T_{\text {marker }} \leftarrow \operatorname{ProxyReEncrypt}\left(C T_{e c}, m r k, m c k_{w}\right)$. The cloud server can run this re-encryption algorithm. On input a ciphertext $C T_{e c}$ and corresponding condition $w$ under public key $h_{e c}^{\prime}$, a partial re-encryption key $m r k$ and a condition key $m c k_{w}$, It outputs a re-encrypted ciphertext $C T_{\text {marker }}$ under a marker's public key $m p k$.

- qcontent $/ \perp \leftarrow \operatorname{CPREDecrypt}\left(C T_{\text {marker }}, m s k\right)$. On input a secret key $m s k$ and a cipertext $C T_{\text {marker }}$. It decrypts the qcontent or the error symbol $\perp$.

- qcontent $/ \perp \leftarrow \operatorname{OrgDecrypt}\left(C T_{e c}, h_{e c}^{\prime}\right)$. On input a secret key $a s k_{e c}$ and a cipertext $C T_{e c}$. It decrypts the qcontent or the error symbol $\perp$.

- $C T_{e c} \leftarrow$ StoreAnswerData(). After EC scans and processes the answer sheet, build a secure local database, encrypts, and stores answer data to the cloud server according to its rules (conditions) to save local storage.

$-\left(C T_{\text {marker }},\{q i d, \sigma\}\right) \leftarrow$ PostAnswerData(). The EC sends instructions to the cloud server to convert the stored ciphertext to a new ciphertext that the specified marker can decrypt and sends to the marker. Besides, to enable the marker to provide acceptable answer data source authentication, the EC needs to send authorization commands to the indicated marker.

- $\left(\left\{\right.\right.$ qid, qcontent $_{n}$, cotpk $\left.\}\right) \leftarrow$ GetAnswerData $\left(C T_{\text {marker }}\right.$, $m s k,\{q i d, \sigma\})$. A marker get $C T_{\text {marker }}$ from cloud server, a set of qid, $\sigma$ from the EC, and decrypts ciphertext by his/her secret key $m s k$.

- (qid, cotpk, $m p k, \sigma) \leftarrow$ PreparetoEndorser(). After running GetAnswerData(), the marker evaluates the content of each answer and gives an evaluation result. The marker sends ( $q i d, \cot p k, m p k, \sigma$ ) and evaluation result to endorsers.

$-1 / 0 \leftarrow$ EndorserVerifyAuth(qid, cotpk, $m p k, \sigma)$. On input the processed data by a marker, it outputs 1 if it is valid and outputs 0 otherwise.

\subsubsection{Security Property}

EC's signature on the candidate and question ID is unforgeable if the discrete logarithm (DL) assumption holds in $\mathbb{G}_{1}$ in RO. Answer content data is secure on the cloud server if the 3-QBDH assumption holds in $\mathbb{G}_{1}, \mathbb{G}_{T}^{\prime}$ in RO.

\subsubsection{Proof Sketch}

Observe that $\sigma=\operatorname{Sign}\left(a_{s k}\right.$, , qid $\left.\|\operatorname{cotp} k\| m p k\right)$. The unforgeable of EC's signature follows from the unforgeability of the BBS [26] under the DL assumption holds in $\mathbb{G}_{1}$ in RO. The security of stored answer content data on cloud server is secure based on the CCA secure C-PRE scheme under the 3 -QBDH assumption holds in $\mathbb{G}_{1}, \mathbb{G}_{T}^{\prime}$ in RO. The security proof of CCA secure refer on [28] (Sect. 4.2).

\subsubsection{Construction Details}

In this part, the examination center distributes the answer data to markers in an end-to-end manner. In our conference version of ExamChain [19], each communication between the two parties can only send one question's answer content for one candidate. The EC stores answer data in a secure local database, which requires very high conditions, such as communication within the local network to prevent data leakage and sizeable local storage. In practical applications, it is not easy not to rely on third-party cloud servers. If cloud services are used, there will be a security risk of data leakage, so we introduced proxy re-encryption technology [29]. The critical data processed by the examination center is stored in the third-party proxy server as ciphertext, and the proxy server cannot obtain the transmitted message plaintext during the whole process. The use of the existing PRE requires that the trusted cloud server can execute the user-specified access control policy. However, this 
trust model is unrealistic in many applications. C-PRE can use higher-trust agents than existing PRE solutions. In addition, through conditional proxy re-encryption [28], multiple answer data can be sent in one communication. For a fair examination evaluation process, candidate and question ID authentication is necessary. We verify the EC's signature to ensure that the data submitted by the marker to the endorser is indeed certified by the EC. Preventing collusion between markers leads to unauthorized markers scoring indiscriminately.

* MarKeyGen A marker picks secret key is $m s k=x \in \mathbb{Z}_{p}^{*}$ and public key is $m p k=\left(P_{1}, P_{2}\right)=\left(g^{x}, g_{1}^{1 / x}\right)$.

* RencKeyGen On input a secret key $a s k_{e c}$ and a public key $m p k=\left(P_{1}, P_{2}\right)=\left(g^{x}, g_{1}^{1 / x}\right)$, it outputs $m r k=$ $P_{1}^{1 / a_{\text {ask }}}=g^{x / a s k_{e c}}$.

* CondKeyGen On input the EC's secret key $a s k_{e c}$ and a condition $w \in\{0,1\}^{*}$, it outputs $m c k_{w}=H_{3}\left(w, h_{e c}^{\prime}\right)^{1 / a s k_{e c}}$ as condition key.

* ProxyEncrypt On input the EC's public key $h_{e c}^{\prime}=$ $\left(P_{1}, P_{2}\right)=\left(g^{a^{a s k_{e c}}}, g_{1}^{1 / a s k_{e c}}\right)$, a condition $w$ and a set of local plaintext answer content qcontent $\in\{0,1\}^{l_{0}}$.

1. Choose $r^{\prime} \stackrel{\$}{\leftarrow}\{0,1\}^{l_{1}}$ and compute $r=$ $H_{1}$ (qcontent, $\left.r^{\prime}, h_{e c}^{\prime}, w\right)$.

2. The ciphertext $C T_{e c}=(A, B, C, D)$ is encrypted as follows:

$$
\begin{aligned}
A= & g_{1}^{r}, \quad B=P_{1}^{r}, \\
C= & H_{2}\left(e(g, g)^{r}\right) \oplus\left(q \text { content } \| r^{\prime}\right) \\
& \oplus H_{4}\left(e\left(P_{2}, H_{3}\left(w, h_{e c}^{\prime}\right)\right)^{r}\right), \\
D= & H_{5}(A, B, C)^{r}
\end{aligned}
$$

* ProxyReEncrypt On input the ciphertext $C T_{e c}$, right condition $w$ under EC's public key, a condition key $m c k_{w}$ and a partial re-encryption key $m r k$, it computes a re-encrypted ciphertext under $m p k$, as shown below:

1. Parse $h_{e c}^{\prime}$ as $\left(P_{1}, P_{2}\right)$ and $C T_{e c}$ as $(A, B, C, D)$.

2 . If the following equation does not hold, then output $\perp$. Otherwise, the re-encrypted ciphertext $C T_{\text {marker }}=\left(B^{\prime}, C^{\prime}\right)$ is calculated as follows:

$$
\begin{aligned}
& e\left(A, P_{1}\right)=e\left(g_{1}, B\right), \\
& e\left(A, H_{5}(A, B, C)\right)=e\left(g_{1}, D\right) . \\
& B^{\prime}=e(B, m r k), \\
& C^{\prime}=C \oplus H_{4}\left(e\left(A, m c k_{w}\right)\right) .
\end{aligned}
$$

* CPREDecrypt On input a secret key msk and a reencrypted ciphertext $C T_{\text {marker }}=\left(B^{\prime}, C^{\prime}\right)$ under public key $m p k=\left(P_{1}, P_{2}\right)$, this algorithm works as follows: First ensure that $m p k \neq h_{e c}^{\prime}$. Then compute (qcontent $\left.\| r^{\prime}\right)=C^{\prime} \oplus H_{2}\left(B^{\prime 1 / a s k_{e c}}\right)$, and return qcontent if $e(g, g)^{a s k_{e c} \cdot H_{1}\left(q \text { content }, r^{\prime}, h_{e c}^{\prime}, w\right)}=B^{\prime}$ holds and $\perp$ otherwise.

* OrgDecrypt On input a secret key $a s k_{e c}$ and an original ciphertext $C T_{e c}$, under public key $h_{e c}^{\prime}=$ $\left(P_{1}, P_{2}\right)$ and condition $w$, this algorithm works as follows: $C T_{e c}=(A, B, C, D)$ : If Eq. (3) does not hold, output $\perp$; otherwise, generates (qcontent $\left.\| r^{\prime}\right)=$ $C \oplus H_{4}\left(e\left(A, H_{3}\left(w, h_{e c}^{\prime}\right)^{1 / x}\right)\right) \oplus H_{2}\left(e(B, g)^{1 / x}\right)$ and return qcontent if $g^{x \cdot H_{1}\left(q c o n t e n t, r^{\prime}, h_{e c}^{\prime}, w\right)}=B$ holds and $\perp$ otherwise.

* StoreAnswerData For example, the EC sets the question number $n$ as a condition $w$, so that multiple data of a certain question $n$ can be packaged and sent to the indicated marker. We assume qcontent equal to a set of (qid\|qcontent $\left\|_{n}\right\|$ cotpk) under condition $n$. Then the EC runs $C T_{e c} \leftarrow \operatorname{ProxyEncrypt}\left(h_{e c}^{\prime}, q c o n t e n t, w\right)$ and sends $C T_{e c}$ to a cloud server.

* PostAnswerData When the EC need to distribute answer content to markers.

- Firstly, the EC picks a marker's public key $m p k$, send a partial re-encryption key $m r k$ to the cloud server by $m r k \leftarrow \operatorname{RencKeyGen}\left(a s k_{e c}, m p k\right)$.

- Secondly, the EC choose a question number $n$ as condition, send the condition key $m c k_{n}$ to the cloud server by $m c k_{n} \leftarrow \operatorname{CondKeyGen}\left(a s k_{e c}, n\right)$.

- Thirdly, the cloud server run $C T_{\text {marker }} \leftarrow$ ProxyReEncrypt $\left(C T_{e c}, m r k, m c k_{w}\right)$ to convert the stored original ciphertext $C T_{e c}$ to a new reencrypted ciphertext $C T_{\text {marker }}$ which can only be decrypted by the indicated marker.

- Finally, in order to enable the marker to provide acceptable answer data source authentication, the EC runs and sends a set of $\sigma=$ $\operatorname{Sign}\left(a_{s k_{e c}}, q i d\|\operatorname{cotpk}\| m p k\right)$ for each question $n$ and marker to the indicated marker.

* GetAnswerData A marker receives data $C T_{\text {marker }}$ from cloud server and a set of $(q i d, \sigma)$ from the EC, then he/she decrypts ciphertext by qcontent $/ \perp \leftarrow$ CPREDecrypt $\left(C T_{\text {marker }}\right.$, msk $)$ and parses qcontent as a set of question ID qid, each answer content qcontent ${ }_{n}$ and candidate's one-time public key cotpk.

* PreparetoEndorser After running GetAnswerData(), the marker evaluates the content of each answer and gives an evaluation result. He/She can send the question ID qid, the one-time pubic key cotpk bound with the candidate to endorsers, his/her public key $m p k$, and $\sigma$ used to verify that the qid comes from the valid EC and is indeed sent to the marker himself/herself. Note that the marker does not send plaintext answer content to anyone.

* EndorserVerifyAuth After the marker running PreparetoEndorser(), the endorser verify whether the question ID and candidate's one-time public key submitted by the marker is issued by the EC. If $1=$ VerifySign $\left(h_{e c}, \sigma\right.$, qid $\left.\|\cot p k\| m p k\right)$ output true, and outputs false otherwise. 


\subsection{Marking Scores Privacy}

\subsubsection{System Model}

Marking scores privacy is essential for extensive public examinations. To ensure the correctness of the examination papers, each score should be within the specified range. To increase the marking process's flexibility to meet more examination scenarios, the marking rules should be automated by peers in the blockchain system.

- $\left(C_{\text {score }}, r_{\text {score }}, C_{\text {score }}^{\prime}, r_{\text {score }}^{\prime}, \pi_{\text {score }}\right) \leftarrow \operatorname{EncProof}\left(m_{\text {score }}\right)$. When a marker submits a score $m_{\text {score }} \in[0, R]$, it outputs $C_{\text {score }}, r_{\text {score }}$ which is encrypted to the EC, $C_{\text {score }}^{\prime}$, $r_{\text {score }}^{\prime}$ which is encrypted to the candidate, and a zeroknowledge proof $\pi_{\text {score }}$ that $m_{\text {score }} \in[0, R]$ in encrypted form.

$-1 / 0 \leftarrow \operatorname{EncVerify}\left(C_{\text {score }}, C_{\text {score }}^{\prime}, \pi_{\text {score }}\right)$. The verifier can varify $\pi_{\text {score }}$ is a valid zero-knowledge proof of $C_{\text {score }}$, $C_{\text {score }}^{\prime}$, and output 1 if true. Otherwise output 0.

- $m_{\text {score }} \leftarrow \operatorname{Decrypt}\left(a k_{e c}, C_{\text {score }}\right) / \operatorname{Decrypt}\left(\operatorname{cotsk}, C_{\text {score }}^{\prime}\right)$. The EC uses secret key $a s k_{e c}$ to decrypt $C_{\text {score }}$, and outputs plaintext score $m_{\text {score }}$. The candidate use one-time secret key cotsk to decrypt $C_{\text {score }}^{\prime}$, and outputs plaintext score $m_{\text {score }}$.

\subsubsection{Security Property}

Our marking score privacy protocol is sound if the u-Strong Diffie-Hellman (SDH) assumption holds in $\left(\mathbb{G}_{1}, \mathbb{G}_{2}\right)$ in RO. The score is secret except for the EC who has EC's secret key and the corresponding candidate who has a one-time secret key if the DDH assumption holds in $\mathbb{G}_{1}$ in RO.

\subsubsection{Proof Sketch}

The soundness follows from the unforgeability of the BBS [26] and the extraction property of the proof of knowledge protocol. The basic BBS scheme is u-secure against an existential forgery under a weak chosen message attack under the q-strong Diffie-Hellman assumption holds in $\left(\mathbb{G}_{1}, \mathbb{G}_{2}\right)$ in $\mathrm{RO}$. The honest-verifier zero-knowledge (HVZK) property follows from the perfect blinding of the signatures in the first phase and the corresponding HVZK property of the sigma protocol. The privacy of the score is straightforward following the IND-CPA security of the underlying ElGamal encryption, and the zero-knowledge property of $\pi_{\text {score }}$ under the DDH assumption holds in $\mathbb{G}_{1}$ in RO.

\subsubsection{Construction Details}

We decompose the K-bit score $m_{\text {score }}$ into $l$ segment $\mu_{0}, \ldots, \mu_{l-1}$ which are smaller than the message space $u$ by $m_{\text {score }}=\sum_{j=0}^{l-1} \mu_{j} u^{j}$. Therefore we encrypt each $\mu_{j}$ by additive homomorphic ElGamal encryption. ECC ElGamal encryption is easy to combine with the Boneh-Boyen signature-based range proof [27] which required a trusted setup.

* EncProof The prover wants to prove $m_{\text {score }}$ lies in $\left[0, u^{l}\right]$. It first decomposes $m_{\text {score }}$ into $\mu_{k} \in[0, u-1]$ such that $m_{\text {score }}=\sum_{k=0}^{l-1} \mu_{k} u^{k}$. Secondly, for each $\mu_{k}$ the prover computes $\left(C_{k}=g_{0}^{\mu_{k}} h_{e c}^{r_{k}}, B_{k}=g^{r_{k}}\right)$ for some ran$\operatorname{dom} r_{k} \in \mathbb{Z}_{p}$ to EC. Denote $C_{\text {score }}=\left\{C_{k}, B_{k}\right\}_{k \in[0, l-1]}$ and $r_{\text {score }}=\sum_{k=0}^{l-1} r_{k} u^{k}$. And computes $\left(C_{k}^{\prime}=g_{0}^{\mu_{k}} \operatorname{cotp}^{r_{k}^{\prime}}\right.$, $B_{k}=g^{r_{k}^{\prime}}$ ) for some random $r_{k}^{\prime} \in \mathbb{Z}_{p}$ to corresponding candidate. Denote $C_{\text {score }}^{\prime}=\left\{C_{k}^{\prime}, B_{k}^{\prime}\right\}_{k \in[0, l-1]}$ and $r_{\text {score }}^{\prime}=\sum_{k=0}^{l-1} r_{k}^{\prime} u^{k}$. Thirdly, for each $\mu_{k}$ it proves that the encrypted $\mu_{k}$ corresponds to some $A_{\mu_{k}}$ :

$$
\begin{aligned}
\pi_{\text {score }} \leftarrow & \operatorname{PoK}\left\{\left(\left\{\mu_{k}, r_{k}, A_{\mu_{k}}\right\}_{k \in[0, l-1]}\right):\right. \\
& \hat{e}(g, \hat{g})=\hat{e}\left(A_{\mu_{k}}, \hat{g}^{\mu_{k}} \cdot \hat{Y}\right) \\
& \wedge C_{k}=g_{0}^{\mu_{k}} h_{e c}^{r_{k}} \wedge B_{k}=g^{r_{k}} \\
& \left.\wedge C_{k}^{\prime}=g_{0}^{\mu_{k}} \operatorname{cotp}^{r^{\prime}} \wedge B_{k}^{\prime}=g^{r_{k}^{\prime}}\right\}
\end{aligned}
$$

It outputs $\left(C_{\text {score }}, r_{\text {score }}, C_{\text {score }}^{\prime}, r_{\text {score }}^{\prime}, \pi_{\text {score }}\right)$.

* EncVerify On input the ciphertext $C_{\text {score }}=\left\{C_{k}, B_{k}\right\}_{k \in[0, l-1]}$, $C_{\text {score }}^{\prime}=\left\{C_{k}^{\prime}, B_{k}^{\prime}\right\}_{k \in[0, l-1]}$ and the proof $\pi_{\text {score }}$, the verifier verify the proof. The details of this protocol can be found in appendix Appendix A. If proof is valid, output 1 or 0 otherwise.

* Decrypt The EC's secret key is $a s k_{e c}$. The encrypted score is $C_{\text {score }}=\left\{C_{k}, B_{k}\right\}_{k \in[0, l-1]}$. The EC computes $g_{0}^{\mu_{k}}=$ $\frac{C_{k}}{B_{k}^{a s t e c}}$ for $k \in[0, l-1]$, and uses a pre-computation table containing $\left(g_{0}^{0}, g_{0}^{1}, \ldots, g_{0}^{u-1}\right)$ to find out the value of $\mu_{k}$. The plaintext score is $m_{\text {score }}=\sum_{k=0}^{l-1} \mu_{k} u^{k}$. Similarly, the candidate use his/her one-time secret key cotsk to decrypt $C_{\text {score }}^{\prime}=\left\{C_{k}^{\prime}, B_{k}^{\prime}\right\}_{k \in[0, l-1]}$ by computing $g_{0}^{\mu_{k}}=$ $\frac{C_{k}^{\prime}}{B_{k}^{\text {crots }}}$ for $k \in[0, l-1]$. In the end, the candidate recovers $m_{\text {score }}=\sum_{k=0}^{l-1} \mu_{k} u^{k}$.

\subsection{Marker Identity Privacy}

\subsubsection{System Model}

- (esk,epk) EndKeyGen(). The endorser generates its secret/public key pair $e s k, e p k$.

- cred $\leftarrow$ CredIssue $\left(e p k, m p k, C_{\text {score }}, C_{\text {score }}^{\prime}, q i d, \cot p k, \sigma\right)$. On input endorser's $e p k$, marker's $m p k$, the encrypted scores $C_{\text {score }}, C_{\text {score }}^{\prime}$, question ID qid, question onetime public key belong to which candidate cotpk and $\sigma$ which can prove qid, cotpk is issued by the EC. The endorser issues the credential cred to the marker.

- $\left(C_{t p}, \pi_{t p}\right) \leftarrow$ CredSign $\left(m s k, m p k\right.$, cred $, m_{s c o r e}, r_{\text {score }}$, $\left.r_{\text {score }}^{\prime}, q i d, \operatorname{cotp} k, \sigma\right)$. On input $m s k, m p k$, cred, the randomness $r_{\text {score }}, r_{\text {score }}^{\prime}$ used to encrypt $m_{\text {score }}$, question ID qid, question one-time public key belong to which candidate cotpk and $\sigma$ which can prove qid, cotpk is issued by the EC. It encrypts $m p k$ to the EA and obtains the ciphertext $C_{t p}$. It generates a zeroknowledge proof $\pi_{t p}$ for the correctness of $C_{t p}$ above, knowing $c r e d, m_{\text {score }}, r_{\text {score }}, m p k$, qid, cotpk such that 
$\left(C_{\text {score }}, r_{\text {score }}, C_{\text {score }}^{\prime}, r_{\text {score }}^{\prime}, \pi_{\text {score }}\right) \leftarrow \operatorname{EncProof}\left(m_{\text {score }}\right)$, cred $=$ CredIssue $\left(\right.$ epk, mpk, $C_{\text {score }}, C_{\text {score }}^{\prime}$, qid, cotpk, $\left.\sigma\right)$.

$-1 / 0 \leftarrow \operatorname{Verify}\left(e p k, C_{t p}, \pi_{t p}\right)$. On input the endorser's public key epk, the marker's ciphertext $C_{t p}$ verifies if $\pi_{t p}$ is a vaild zero-knowledge proof. If true, output 1 , and outputs 0 otherwise.

$-m p k \leftarrow \operatorname{Decrypt}\left(a s k_{e a}, C_{t p}\right)$. The EA can use secret key $a s k_{e a}$ to decrypt $C_{t p}$, and get the real indentity of marker $m p k$.

\subsubsection{Security Property}

The marker identity can only be decrypted by the EA. Our marker identity privacy protocol is sound if the q-SDH assumption holds in $\left(\mathbb{G}_{1}, \mathbb{G}_{2}\right)$ in RO. It is unforgeable if the DL assumption holds in $\mathbb{G}_{1}$ in RO. Besides, it is anonymous if the DDH assumption holds in $\mathbb{G}_{1}$ in RO.

\subsubsection{Proof Sketch}

The soundness follows from the unforgeability of the BBS [26] on $\hat{e}\left(Q, E \hat{g}_{2}^{t}\right)=\hat{e}\left(h_{s} g_{1}^{w} g^{m_{\text {score }}} h_{e c}^{r_{\text {score }}} g^{m_{\text {score }}}\right.$ cotp $p k^{r_{\text {score }}^{\prime}}$ cotpkg qid $\left.g^{x}, \hat{g}_{2}\right)$ and the extraction property of the proof of knowledge protocol under the q-SDH assumption holds in $\left(\mathbb{G}_{1}, \mathbb{G}_{2}\right)$ in RO. The unforgrability of the protocol follows from the uncomputability of $P_{1}$ without marker's secret key $x$ under the DL assumption holds in $\mathbb{G}_{1}$ in RO. The marker anonymity is straightforward following the INDCPA security of the underlying ElGamal encryption $C_{t p}=$ $\left(C_{\text {cred }}=P_{1} \cdot h_{\text {ea }}^{r_{\text {cred }}}, B_{\text {cred }}=g^{r_{\text {cred }}}\right)$ and the zero-knowledge property of $\pi_{t p}$ under the DDH assumption holds in $\mathbb{G}_{1}$ in RO. Our marker identity privacy protocol is modified from PAChain [23]. The security proof of soundness, unforgeability, and anonymity is similar to the sender's privacy in PAChain (Appendix B.3).

\subsubsection{Construction Details}

We use anonymous credentials to achieve marker identity privacy and set the honest-but-curious endorser as the group manager.

* EndKeyGen An endorser randomly picks $\alpha \in \mathbb{Z}_{p}$ as secret key esk and computes $E=\hat{g}_{2}^{\alpha}$ as public key epk.

* Credlssue On input an endorser's public key epk, a marker's public key mpk, the ciphertext of the scores $C_{\text {score }}, C_{\text {score }}^{\prime}$, question ID qid, question onetime public key belong to which candidate cotpk and $\sigma$ which can prove qid, cotpk is issued by the EC. Firstly, the marker produce a zero-knowledge proof as $\pi_{\text {marker }}$ of his/her secret key: $x=\log _{g} P_{1}$. $\pi_{\text {score }}$ is the zero-knowledge scores privacy proof (showing the knowledge of $\left(m_{\text {score }}, r_{\text {score }}, r_{\text {score }}^{\prime}\right)$ such that $C_{\text {score }}=g^{m_{\text {score }}} h_{\text {ec }}^{r_{\text {score }}}, C_{\text {score }}^{\prime}=g^{m_{\text {score }}}$ cotp $\left.k^{r_{\text {score }}^{\prime}}\right)$. The marker sends $\pi_{\text {marker }}, \pi_{\text {score }}, \sigma$ to the endorser. In addition, the endorser validates the $\sigma$ by running $1 / 0 \leftarrow$
EndorserVerifyAuth (qid, cotpk, $m p k, \sigma)$. After the endorser validates $\pi_{\text {marker }}, \pi_{\text {score }}$ and $\sigma$, the endorser randomly chooses $w, t \in \mathbb{Z}_{p}$ and compute: $Q=\left(h_{s} \cdot g_{1}^{w}\right.$. $\left.C_{\text {score }} \cdot C_{\text {score }}^{\prime} \cdot \operatorname{cotpk} \cdot g^{q i d} \cdot P_{1}\right)^{\frac{1}{\alpha+t}}$. The endorser issues the credential cred $=(Q, w, t)$ to the marker.

* CredSign The marker inputs tuples $x, m_{\text {score }}, r_{\text {score }}$, $r_{\text {score }}^{\prime}, P_{1}$, qid, cotpk, $Q, C_{\text {score }}, C_{\text {score }}^{\prime}$. Firstly, it encrypts the marker's public key $P_{1}$ to the EA, by randomly choosing $r_{\text {cred }} \in \mathbb{Z}_{p}$ and computing $C_{t p}=\left(C_{\text {cred }}=\right.$ $\left.P_{1} \cdot h_{\text {ea }}^{r_{\text {cred }}}, B_{\text {cred }}=g^{r_{\text {cred }}}\right)$. Secondly, it computes the zero knowledge proof $\pi_{t p}$ for cred $=(Q, w, t)$ corresponds to $P_{1}=g^{x}, C_{\text {score }}=g^{m_{\text {score }}} h_{\text {ec }}^{r_{\text {score }}}, C_{\text {score }}^{\prime}=g^{m_{\text {score }}}$ cotpk $^{r_{\text {score }}^{\prime}}$, qid, cotpk is issued by EC to the indicated marker, and $P_{1}$ is encrypted to the EA.

$$
\begin{aligned}
\pi_{t p}= & \operatorname{PoK}\left\{\left(x, m_{\text {score }}, r_{\text {score }}, r_{\text {score }}^{\prime}, Q, w, t, r_{\text {cred }}, q i d\right):\right. \\
& \hat{e}\left(Q, E \hat{g}_{2}^{t}\right)=\hat{e}\left(h_{s} g_{1}^{w} g^{m_{\text {score }}} h_{\text {ec }}^{r_{\text {score }}} g^{m_{\text {score }}}\right. \\
& \left.\operatorname{cotp} k^{r_{\text {score }}^{\prime}} \operatorname{cotpkg} g^{q i d} g^{x}, \hat{g}_{2}\right) \\
& \wedge C_{\text {cred }}=g^{x} \cdot h_{\text {ea }}^{r_{\text {cred }}} \wedge B_{\text {cred }}=g^{r_{\text {cred }}} \\
& \wedge B_{k}=g^{r_{k}} \wedge C_{k}=g_{0}^{\mu_{k}} \operatorname{cotpk}^{r_{k}} \\
& \left.\wedge B_{k}^{\prime}=g^{r_{k}^{\prime}} \wedge C_{k}^{\prime}=g_{0}^{\mu_{k}} \operatorname{cotpk}^{r_{k}^{\prime}}\right\} .
\end{aligned}
$$

The output $\pi_{t p}, C_{t p}$. The details of zero-knowledge proof is in appendix Appendix B.

* Verify The verifier use endorser's public keys epk to check $\pi_{t p}, C_{t p}$, and checks the validity of the proof $\pi_{t p}$.

* Decrypt The education authority can use its secret key $a s k_{e a}$ to computes the real identity of marker $P_{1}^{\prime}=$ $C_{\text {cred }} / B_{\text {cred }}^{\text {ask }}$.

\section{Performance Analysis}

In our scheme, the two main overheads in performance are range proof [27] and conditional proxy re-encryption [28]. We have analyzed the performance of these two cryptographic protocols and show our scheme is feasible. We use Miracl library to estimate each computing time on Windows 7 operation system using i7-6700U, $3.40 \mathrm{GHz}$ processor and $16 \mathrm{~GB}$ memory. In order to achieve the system security level, we adopts the Barreto Naehrig (BN) curve on 256 for evaluation of range proof. Therefore, the element lengths on $\mathbb{G}_{1}$, $\mathbb{G}_{2}, \mathbb{G}_{T}$, and $\mathbb{Z}$ are 64 bytes, 128 bytes, 384 bytes, and 32 bytes, respectively. We use super-singular curve over $G F(p)$ (512-bit modulus $p, k=2$ ) for evaluation of conditional proxy re-encryption. Here $u$ and $l$ are respectively expressed as base and power of secret value. According to real application, we set $u=10$ and $l=2$ for estimating. $l_{1}$ denotes the security parameter used in Weng et al.'s scheme [28].

We separately count the types and times of operations required by the two protocols, and apply the running time results in Table 1 to calculate the computational cost of each algorithm at each phase, as shown in Table 2 and Table 3. The experimental results show that the performance of the cryptographic protocol can meet the needs of current blockchain applications. 
Table 1 Symbol description and executing time

\begin{tabular}{|c|l|l|}
\hline Notation & Description & Time $(\mathrm{ms})$ \\
\hline$T_{g 1 m}$ & the cost of a point multiplication on $\mathbb{G}_{1}$ & 0.2113 \\
\hline$T_{t m}$ & the cost of a modular multiplication on $\mathbb{G}_{T}$ & 0.6029 \\
\hline$T_{n m}$ & the cost of a modular multiplication on $\mathbb{Z}_{p}^{*}$ & 0.0105 \\
\hline$T_{t m e}$ & the cost of a modular exponentiation on $\mathbb{G}_{T}$ & 0.4322 \\
\hline$T_{m i}$ & the cost of a modular inversion on $\mathbb{Z}_{p}^{*}$ & 0.0455 \\
\hline$T_{g 1 a}$ & the cost of a point addition on $\mathbb{G}_{1}$ & 0.0888 \\
\hline$T_{e t}$ & the cost of a bilinear pairing on $\mathbb{G}_{T}$ & 1.9827 \\
\hline$T_{p}$ & the cost of a bilinear pairing & 24.2309 \\
\hline$T_{e}$ & the cost of an exponentiation & 0.6681 \\
\hline
\end{tabular}

Table 2 Performance analysis of the range proof

\begin{tabular}{|c|l|l|l|}
\hline Types & Phase & Cost & Time(ms) \\
\hline $\begin{array}{c}\text { Communic- } \\
\text { ation } \\
\text { cost }\end{array}$ & Setup & $(u+1)\left|\mathbb{G}_{1}\right|$ & $\backslash$ \\
\cline { 2 - 4 } & Proof & $\begin{array}{c}(2 l+2)\left|Z_{p}\right|+ \\
(l+1)\left|\mathbb{G}_{1}\right|+l\left|\mathbb{G}_{T}\right|\end{array}$ & $\backslash$ \\
\hline $\begin{array}{c}\text { Computation } \\
\text { cost }\end{array}$ & Setup & $(u+1) T_{g 1 m}+u T_{m i}$ & 2.7793 \\
\cline { 2 - 4 } & Prover & $\begin{array}{c}(l+2) T_{g 1 m}+(l+1) T_{e t} \\
+l T_{t m}+2 l T_{t m e} \\
+T_{g 1 a}+(3 l+1) T_{n m}\end{array}$ & 9.8902 \\
\cline { 2 - 4 } & Verifier & $\begin{array}{c}3 T_{g 1 m}+2 l T_{t m} \\
+3 l T_{t m e}+(2 l+1) T_{e t} \\
+n m+2 T_{g 1 a}\end{array}$ & 15.7508 \\
\hline
\end{tabular}

Table 3 Performance analysis of the conditional proxy re-encryption

\begin{tabular}{|l|l|l|l|}
\hline Types & Phase & Cost & Time(ms) \\
\hline \multirow{4}{*}{$\begin{array}{c}\text { Communic- } \\
\text { ation } \\
\text { cost }\end{array}$} & 2nd-level ciphertext & $3|\mathbb{G}|+1|M|+l_{1}$ & $\backslash$ \\
\cline { 2 - 4 } & 1 st-level ciphertext & $1\left|\mathbb{G}_{T}\right|+1|M|+l_{1}$ & $\backslash$ \\
\cline { 2 - 4 } & Public key & $2|\mathbb{G}|$ & $\backslash$ \\
\cline { 2 - 4 } & Priviate key & $1\left|\mathbb{Z}_{p}\right|$ & $\backslash$ \\
\cline { 2 - 4 } & Re-encryption key & $2|\mathbb{G}|$ & \multicolumn{1}{|c|}{$\mid$} \\
\hline \multirow{3}{*}{$\begin{array}{c}\text { Computation } \\
\text { cost }\end{array}$} & Encrypt & $1 T_{p}+5 T_{e}$ & 24.5650 \\
\cline { 2 - 4 } & ReEncrypt & $3 T_{p}+2 T_{e}$ & 72.8263 \\
\cline { 2 - 4 } & Decrypt 2nd-level & $4 T_{p}+5 T_{e}$ & 97.2577 \\
\cline { 2 - 4 } & Decrypt 1st-level & $2 T_{e}$ & 0.1336 \\
\hline
\end{tabular}

\section{Conclusion}

In this paper, we apply the consortium blockchain to OSM. We propose a privacy-preserving, authenticated, and auditable ExamChain, a blockchain-based decentralized marking system for examination. We have altered the traditional fully centralized marking process so that candidates can also trace their scores for each question. The primary motivation of ExamChain is to deploy a decentralized architecture that can audit the marker identity and allow candidates to verify their scores in a distributed access control system under the assumption of a well-defined threat model. We add a semihonest cloud server to store and distribute the enormous answer content data relevant to reality. The blockchain-based architecture provides a strong guarantee of data integrity and availability.

We have put forward the proposal at a high-level. The blockchain-based education information application scheme designed in this article is supposed to have high security and robustness and thus has potential theoretical and application value for the combination of blockchain and the education industry.

\section{Acknowledgments}

This work has been partly supported by the National Natural Science Foundation of China under Grant No.61702212, the Fundamental Research Funds for the Central Universities under Grant No.CCNU19TS017 and the Fundamental Research Funds for the Central University (Innovative Funding projects) under Grant No.2020CXZZ096.

\section{References}

[1] C. Lee, "The role of the hong kong examinations and assessment authority," in Validating Technological Innovation, pp.9-21, Springer Singapore, 2016.

[2] D. Coniam, "Validating onscreen marking in hong kong," Asia Pacific Education Review, vol.11, no.3, pp.423-431, Jan. 2010.

[3] HKEAA, "Hong kong examinations and assessment authority," https://obs.hkeaa.edu.hk/obs/obsindex.jsp, 2021.

[4] DRS, "Drs data services limited home page," http://www.drs.co.uk/ about-us/, 2021.

[5] RMResults, "Rm results home page," https://rmresults.com/, 2021.

[6] DRS, "Drs technology helps zimsec to save costs and ensure that results are published to the candidates in a timely manner." https://drs.co.uk/knowledge-centre/case-studies/case-study/ ZimSEC_case_study, 2015.

[7] DRS, "India's technical university jntu-k automates exam marking with drs," https://drs.co.uk/knowledge-centre/case-studies/casestudy/india-s-technical-university-jntu-k-automates-exam-markingwith-drs, 2014.

[8] M. Thomson, "Ican safeguards students' results in west africa with the help of rm results," https://blog.rmresults.com/news/icansafeguards-students-results-in-west-africa-with-the-help-of-rmresults, 2019.

[9] M. Thomson, "Rm results chosen by nzqa for e-marking of ncea exams," https://blog.rmresults.com/news/rm-results-chosen-by-nzqafor-e-marking-of-ncea-exams, 2019.

[10] RMResults, "Discover how acca doubled exam capacity with emarking," https://content.rmresults.com/acca-doubles-examcapacity-with-e-marking, 2017.

[11] S. Nakamoto, "Bitcoin: A peer-to-peer electronic cash system," https://bitcoin.org/bitcoin.pdf, 2008.

[12] S. Noether, "Ring signature confidential transactions for monero," Cryptology ePrint Archive, Report 2015/1098, https://eprint. iacr.org/2015/1098, 2015.

[13] E.B. Sasson, A. Chiesa, C. Garman, M. Green, I. Miers, E. Tromer, and M. Virza, "Zerocash: Decentralized anonymous payments from bitcoin,” 2014 IEEE Symposium on Security and Privacy, IEEE, May 2014.

[14] C. Garman, M. Green, and I. Miers, "Accountable privacy for decentralized anonymous payments," in Financial Cryptography and Data Security, pp.81-98, Springer Berlin Heidelberg, 2017.

[15] H. Wang and Y. Song, "Secure cloud-based EHR system using attribute-based cryptosystem and blockchain," Journal of Medical Systems, vol.42, no.8, July 2018.

[16] S. Wang, Y. Zhang, and Y. Zhang, "A blockchain-based framework for data sharing with fine-grained access control in decentralized storage systems," IEEE Access, vol.6, pp.38437-38450, 2018.

[17] J. Liu, X. Li, L. Ye, H. Zhang, X. Du, and M. Guizani, "BPDS: A blockchain based privacy-preserving data sharing for electronic medical records," 2018 IEEE Global Communications Conference (GLOBECOM), IEEE, Dec. 2018.

[18] E. Androulaki, A. Barger, V. Bortnikov, C. Cachin, K. Christidis, A.D. Caro, D. Enyeart, C. Ferris, G. Laventman, Y. Manevich, S. 
Muralidharan, C. Murthy, B. Nguyen, M. Sethi, G. Singh, K. Smith, A. Sorniotti, C. Stathakopoulou, M. Vukolić, S.W. Cocco, and J. Yellick, "Hyperledger fabric," Proc. Thirteenth EuroSys Conference, ACM, April 2018.

[19] H. An and J. Chen, "Examchain: A privacy-preserving onscreen marking system based on consortium blockchain," 2021 IEEE Conference on Dependable and Secure Computing (DSC), pp.1-8, 2021.

[20] E. Androulaki, G.O. Karame, M. Roeschlin, T. Scherer, and S. Capkun, "Evaluating user privacy in bitcoin," in Financial Cryptography and Data Security, pp.34-51, Springer Berlin Heidelberg, 2013.

[21] N. van Saberhagen, "Cryptonote v 2.0," 2013.

[22] Y. Li, W. Susilo, G. Yang, Y. Yu, X. Du, D. Liu, and N. Guizani, "Toward privacy and regulation in blockchain-based cryptocurrencies," IEEE Netw., vol.33, no.5, pp.111-117, Sept. 2019.

[23] T.H. Yuen, "PAChain: Private, authenticated and auditable consortium blockchain," in Cryptology and Network Security, pp.214-234, Springer International Publishing, 2019.

[24] S. Shi, D. He, L. Li, N. Kumar, M.K. Khan, and K.-K.R. Choo, "Applications of blockchain in ensuring the security and privacy of electronic health record systems: A survey," Computers \& Security, vol.97, p.101966, 2020.

[25] Y. Sun, R. Zhang, X. Wang, K. Gao, and L. Liu, "A decentralizing attribute-based signature for healthcare blockchain," 2018 27th International Conference on Computer Communication and Networks (ICCCN), IEEE, July 2018.

[26] D. Boneh and X. Boyen, "Short signatures without random oracles," in Advances in Cryptology - EUROCRYPT 2004, pp.56-73, Springer Berlin Heidelberg, 2004.

[27] J. Camenisch, R. Chaabouni, and abhi shelat, "Efficient protocols for set membership and range proofs," in Advances in Cryptology ASIACRYPT 2008, pp.234-252, Springer Berlin Heidelberg, 2008.

[28] J. Weng, R.H. Deng, X. Ding, C.-K. Chu, and J. Lai, "Conditional proxy re-encryption secure against chosen-ciphertext attack," Proc. 4th International Symposium on Information, Computer, and Communications Security - ASIACCS '09, ACM Press, 2009.

[29] R. Canetti and S. Hohenberger, "Chosen-ciphertext secure proxy re-encryption," Proc. 14th ACM conference on Computer and communications security - CCS '07, ACM Press, 2007.

\section{Appendix A: Protocol to Prove Marking Scores Pri- vacy}

This section provides the details of the protocol denoted.

$$
\begin{aligned}
\pi_{\text {score }} \leftarrow & \operatorname{PoK}\left\{\left(\left\{\mu_{k}, r_{k}, A_{\mu_{k}}\right\}_{k \in[0, l-1]}\right):\right. \\
& \hat{e}(g, \hat{g})=\hat{e}\left(A_{\mu_{k}}, \hat{g}^{\mu_{k}} \cdot \hat{Y}\right) \\
& \wedge C_{k}=g_{0}^{\mu_{k}} h_{e c}^{r_{k}} \wedge B_{k}=g^{r_{k}} \\
& \left.\wedge C_{k}^{\prime}=g_{0}^{\mu_{k}} \cot ^{\mu^{\prime}} k^{r_{k}^{\prime}} \wedge B_{k}^{\prime}=g^{r_{k}^{\prime}}\right\}
\end{aligned}
$$

that can be used (as described in Sect. 5.3) to prove that each $\mu_{k}$ corresponds to some $A_{\mu_{k}}$.

(1) The prover randomly picks $v_{k}, s_{k}, t_{k}, v \in \mathbb{Z}_{p}$ for $k \in[0, l-1]$ and computes:

$$
\begin{aligned}
& V_{k}=A_{\mu_{k}}^{v_{k}}, \quad a_{k}=\hat{e}\left(V_{k}, \hat{g}\right)^{-s_{k}} \cdot \hat{e}(g, \hat{g})^{t_{k}}, \\
& E_{k}=g_{0}^{s_{k}} h_{e c}^{v_{k}}, \quad E_{k}^{\prime}=g_{0}^{s_{k}} \operatorname{cotpk}^{v_{k}}, \quad D_{k}=g^{v_{k}} .
\end{aligned}
$$

(2) It computes $\tilde{c}=H\left(\right.$ param,$\left\{V_{k}, a_{k}, B_{k}, C_{k}, B_{k}^{\prime}, C_{k}^{\prime}, D_{k}, E_{k}\right.$, $\left.\left.E_{k}^{\prime}\right\}_{k \in[0, l-1]}\right)$ and for $k \in[0, l-1]$ :

$$
z_{\mu_{k}}=s_{k}-\tilde{c} \mu_{k}, \quad z_{v_{k}}=t_{k}-\tilde{c} v_{k}, \quad z_{r_{k}}=v_{k}-\tilde{c} r_{k} .
$$

Then $\pi_{\text {score }}=\left(\left\{V_{k}, z_{\mu_{k}}, z_{v_{k}}, z_{r_{k}}\right\}_{k \in[0, l-1]}, \tilde{c}\right)$. It outputs $\left(C_{\text {score }}, r_{\text {score }}, C_{\text {score }}^{\prime}, r_{\text {score }}^{\prime}, \pi_{\text {score }}\right)$.

(3) The verifier inputs the ciphertext $C_{\text {score }}=\left\{C_{k}, B_{k}\right\}_{k \in[0, l-1]}$, $C_{\text {score }}^{\prime}=\left\{C_{k}^{\prime}, B_{k}^{\prime}\right\}_{k \in[0, l-1]}$ and the proof $\pi_{\text {score }}=$ $\left(\left\{V_{k}, z_{\mu_{k}}, z_{v_{k}}, z_{r_{k}}\right\}_{k \in[0, l-1]}, \tilde{c}\right)$, the verifier computes for all $k \in[0, l-1]$.

$$
\begin{aligned}
& D_{k}=B_{k}^{\tilde{c}} g^{z_{r_{k}}}, \quad E_{k}=C_{k}^{\tilde{c}} g_{0}^{z_{\mu_{k}}} h_{e c}^{z_{r_{k}}}, \\
& E_{k}^{\prime}=C_{k}^{\prime \tilde{c}} g_{0}^{z_{\mu_{k}}} \cot ^{z^{r_{k_{k}}}}, \\
& a_{k}=\hat{e}\left(V_{k}, \hat{Y}^{\tilde{c}} \hat{g}^{-z_{\mu_{k}}}\right) \cdot \hat{e}(g, \hat{g})^{z_{v_{k}}}
\end{aligned}
$$

If $\tilde{c}=H\left(\right.$ param, $\left.\left\{V_{k}, a_{k}, B_{k}, C_{k}, B_{k}^{\prime}, C_{k}^{\prime}, D_{k}, E_{k}, E_{k}^{\prime}\right\}_{k \in[0, \ell-1]}\right)$, output 1 or 0 otherwise.

\section{Appendix B: Protocol to Prove Marker Identity Pri- vacy}

This section provides the details of the protocol denoted

$$
\begin{aligned}
\pi_{t p}= & \operatorname{PoK}\left\{\left(x, m_{\text {score }}, r_{\text {score }}, r_{\text {score }}^{\prime}, Q, w, t, r_{\text {cred }}, q i d\right):\right. \\
& \hat{e}\left(Q, E \hat{g}_{2}^{t}\right)=\hat{e}\left(h_{s} g_{1}^{w} g^{m_{\text {score }}} h_{e c}^{r_{\text {score }}} g^{m_{\text {score }}}\right. \\
& \left.\operatorname{cotp} p k^{r_{\text {score }}} \cot p k g^{q i d} g^{x}, \hat{g}_{2}\right) \\
& \wedge C_{\text {cred }}=g^{x} \cdot h_{\text {cead }}^{r_{\text {cred }}} \wedge B_{\text {cred }}=g^{r_{\text {cred }}} \\
& \wedge B_{k}=g^{r_{k}} \wedge C_{k}=g_{0}^{\mu_{k}} \operatorname{cotp} k^{r_{k}} \\
& \left.\wedge B_{k}^{\prime}=g^{r_{k}^{\prime}} \wedge C_{k}^{\prime}=g_{0}^{\mu_{k}} \cot p k^{r_{k}^{\prime}}\right\} .
\end{aligned}
$$

(1) The prover picks $r_{\psi}, r_{a}, r_{b}, r_{k}, r_{t}, r_{m}, r_{r}, r_{w} \in \mathbb{Z}_{p}, a$ randomly. It computes:

$$
\begin{aligned}
& S=Q \cdot u_{1}^{a}, \\
& \begin{array}{l}
\xi=g_{1}^{a}, R_{\text {cred }, 1} \\
\quad=\hat{e}\left(u_{1}^{r_{b}} S^{-r_{t}} g_{1}^{r_{w}} g^{r_{m}} h_{e a}^{r_{r}} g^{r_{k}}, \hat{g}_{2}\right) \cdot \hat{e}\left(u_{1}, E\right)^{r_{a}}, \\
R_{\text {cred }, 2}=g_{1}^{r_{a}}, \quad R_{\text {cred }, 3}=\xi^{r_{t}} g_{1}^{-r_{b}}, \\
R_{\text {cred }, 4}=g^{r_{\psi}}, \quad R_{\text {cred }, 5}=g^{r_{k}} h_{e a}^{r_{\psi}} .
\end{array}
\end{aligned}
$$

(2) The prover computes $c=H\left(R_{\text {cred }, 1}, R_{\text {cred }, 2}, R_{\text {cred }, 3}\right.$, $\left.R_{\text {cred }, 4}, R_{\text {cred }, 5}, C_{t p}, S, \xi\right)$.

(3) The prover computes:

$$
\begin{aligned}
& z_{k}=r_{k}+c \cdot x^{\prime}, \quad z_{b}=r_{b}+c \cdot a \cdot z, \\
& z_{r}=r_{r}+c \cdot r^{\prime}, \quad z_{a}=r_{a}+c \cdot a, \\
& z_{w}=r_{w}+c \cdot w, \quad z_{\psi}=r_{\psi}+c \cdot r_{c r e d}, \\
& z_{t}=r_{t}+c \cdot t, \quad z_{m}=r_{m}+c \cdot m^{\prime} .
\end{aligned}
$$

Outputs the proof $\pi_{t p}=\left(c, S, \xi, z_{k}, z_{a}, z_{t}, z_{b}, z_{w}, z_{m}, z_{r}, z_{\psi}\right)$. (4) The verifier receives $\pi_{t p}$ and computes:

$$
\begin{aligned}
& R_{c r e d, 1}^{\prime}=\hat{e}\left(u_{1}^{z_{b}} S^{-z_{t}} g_{1}^{z_{w}} g^{z_{m}} h_{e a}^{z_{r}} g_{1}^{z_{k}} h_{s}^{c}, \hat{g}_{2}\right) \cdot \hat{e}\left(u_{1}^{z_{a}} S^{-c}, E\right), \\
& R_{c r e d, 2}^{\prime}=g_{1}^{z_{a}} \xi^{-c}, \quad R_{c r e d, 3}^{\prime}=\xi^{z_{t}} g_{1}^{-z_{b}}, \\
& R_{\text {cred }, 4}^{\prime}=g^{z_{\psi}} B_{\text {cred }}^{-c}, \quad R_{\text {cred }, 5}^{\prime}=g^{z_{k}} h_{e a}^{z_{\psi}} C_{c r e d}^{-c} .
\end{aligned}
$$

(5) The verifier computes $c^{\prime}=H\left(R_{\text {cred }, 1}, R_{\text {cred }, 2}, R_{\text {cred }, 3}\right.$, $\left.R_{\text {cred, } 4}, R_{c r e d, 5}, C_{t p}, S, \xi\right)$. It outputs 1 if $c=c^{\prime}$, and outputs 0 otherwise. 


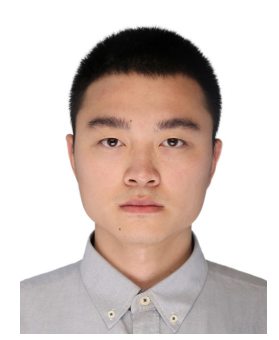

Haoyang An received his B.S. degree in information security from Hefei University of Technology (HFUT) in 2019, and received his M.S. of computer science from the Wollongong Joint Institute, Central China Normal University (CCNU) and University of Wollongong (UOW) in 2021. He is currently a PHD candidate at Wuhan University. His research interests include cryptography and blockchain.

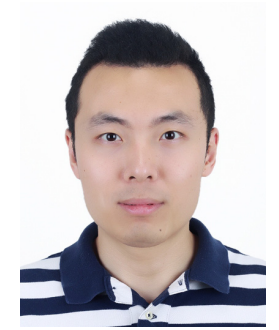

Jiageng Chen received the B.S. degree from the School of Computer Science and Technology, Huazhong University of Science and Technology (HUST) in 2004 and received his M.S. and Ph.D. of computer science from the School of Information Science, Japan Advanced Institute of Science and Technology (JAIST) in 2007 and 2012, respectively. He was working as an Assistant Professor in School of Information Science, Japan Advanced Institute of Science and Technology from 2012 to 2015. And currently, he is an Associate Professor at the Computer School of Central China Normal University. His research areas include cryptography, especially in the areas of algorithms, cryptanalysis, secure designs, and so on. 